\title{
MiR-29c inhibits the metastasis of oral squamous cell carcinoma and promotes its cell cycle arrest by targeting SERPINH1
}

\author{
Chuanning Wang, Zhiming Wang, Liping Zhang, Xiaoping Lin \\ Department of Stomatology, Shengjing Hospital of China Medical University, Shenyang, China \\ Contributions: (I) Conception and design: C Wang; (II) Administrative support: X Lin; (III) Provision of study materials or patients: Z Wang, \\ L Zhang; (IV) Collection and assembly of data: C Wang, X Lin; (V) Data analysis and interpretation: C Wang, X Lin; (VI) Manuscript writing: All \\ authors; (VII) Final approval of manuscript: All authors. \\ Correspondence to: Xiaoping Lin. Department of Stomatology, Shengjing Hospital of China Medical University, 36 Sanhao Street, Shenyang 110004 , \\ China. Email: linxp@sj-hospital.org.
}

\begin{abstract}
Background: A large number of studies have shown that the imbalance of micro RNA (miRNA) and its target genes can promote the development of tumors. The purpose of this study was to investigate the biological role and molecular mechanism of serpin peptidase inhibitor clade H member 1 (SERPINH1) and its upstream regulator miR-29c in oral squamous cell carcinoma (OSCC).

Methods: The expression levels of SERPINH1 and miR-29c were detected by quantitative reverse transcription polymerase chain reaction (RT-qPCR) and western blotting. The proliferation, apoptosis, metastasis, and cell cycle were detected by 3-(4,5-dimethylthiazol-2-yl)-2,5-diphenyl tetrazolium bromide (MTT) assay, would healing assay, transwell assay, flow cytometry, and dual luciferase reporter assay.

Results: High expression of SERPINH1 was detected in patients with OSCC and it can be used as a prognostic biomarker for OSCC. Cell function experiments showed that silencing the expression of SERPINH1 inhibited the proliferation and migration of OSCC cells and caused cell cycle arrest at $\mathrm{S}$ phase. Bioinformatics analysis showed that there was a binding site between miR-29c and SERPINH1, indicating that miR-29c may regulate the expression of SERPINH1. In addition, miR-29c overexpression inhibited the proliferation and metastasis of OSCC cells, and the subsequent rescue experiment showed that SERPINH1 overexpression can reverse the inhibitory effect of miR-29c in OSCC cell proliferation, migration, apoptosis, and cell cycle arrest.
\end{abstract}

Conclusions: The miRNA, miR-29c can regulate the proliferation, migration, invasion, and cell cycle of OSCC cells by targeting SERPINH1.

Keywords: Oral squamous cell carcinoma (OSCC); serpin peptidase inhibitor clade H member 1 (SERPINH1); miR-29c; proliferation; metastasis

Submitted Jun 28, 2021. Accepted for publication Sep 02, 2021.

doi: $10.21037 /$ atm-21-3720

View this article at: https://dx.doi.org/10.21037/atm-21-3720

\section{Introduction}

In 2018, there were an estimated 800,000 new cases of head and neck squamous cell carcinoma (HNSCC) and more than 450,000 deaths. Classification of HNSCC originating from the mucosa of the upper respiratory tract is made according to location: laryngeal cancer, hypopharyngeal cancer, tonsil cancer, oropharyngeal cancer, and oral cancer (1). Among them, oral cancer is a major public health problem across the world, ranking sixth among human malignancies, with a 5 -year mortality rate of about $50 \%$. Oral malignancies include cancers that occur in the mouth, lips, and pharynx, and oral squamous cell carcinoma (OSCC) accounts for over $90 \%$ of oral cancer. In 2018, OSCC comprised $43.8 \%$ of new HNSCC cases and $37.8 \%$ of deaths (2). Oral cancer is characterized by concealed onset, difficult diagnosis, rapid development, and is often accompanied by metastasis and 
destructive treatment (3). Therefore, it is necessary to find and develop new accurate diagnostic and effective treatment strategies for OSCC.

Serpin peptidase inhibitor clade $\mathrm{H}$ member 1 (SERPINH1), also known as heat shock protein 47 (HSP47), belongs to the Serpin superfamily and is functionally involved in the proper folding and secretion of collagen. The unique characteristics of SERPINH1 in regulating collagen production and its location on the cell membrane of many cancers have led to the utilization of SERPINH1 as a potential biomarker or therapeutic target for many diseases and cancers (4). The abnormal expression of SERPINH1 is closely related to a variety of cancers. Increased expression of SERPINH1 can promote cancer progression and is associated with poor survival, such as in hepatocellular carcinoma (5), lung cancer (6), renal cell carcinoma (7), and gastric cancer (8). Comprehensive screening of proteomics and transcriptome showed that SERPINH1 is strongly correlated with the poor prognosis of renal clear cell carcinoma, and it can regulate the expression of epithelial-mesenchymal transition (EMT) markers (9). Our current The Cancer Genome Atlas (TCGA) analysis showed that SERPINH1 is abnormally expressed in OSCC, but its specific regulatory mechanism is still unclear.

In this study, bioinformatics technology was used to analyze and screen the differentially expressed genes in oral squamous cell carcinoma compared with normal tongue mucosal tissue in TCGA database, and it was found for the first time that SERPINH1 may play a key role in the occurrence and development of OSCC. Through molecular biology experiments, at the cellular level, we found that silencing SERPINH1 could inhibit the growth and migration of OSCC cells, and SERPINH1 may be regulated by miR29c. Therefore, exploring the molecular mechanism of SERPINH1 in OSCC will help us further understand the pathogenesis of OSCC. We present the following article in accordance with the MDAR reporting checklist (available at https://dx.doi.org/10.21037/atm-21-3720).

\section{Methods}

\section{Bioinformatics}

The GSE31056 microarray dataset containing gene expression data of 23 OSCC tumors and their normal marginal tissues was downloaded from the Gene Expression Omnibus (GEO) database (https://www.ncbi.nlm.nih. gov/geo/). The differentially expressed messenger RNAs (mRNAs) between normal group and tumor group in GSE3 1056 were obtained by the R language analysis tool GEO2R (10). At the same time, the public cancer transcriptome database UALCAN (http://ualcan.path. uab.edu/index.html) was used to examine the expression and patient survival information of SERPINH1, while TargetScan (http://www.targetscan.org/vert_72/) was used to identify the putative upstream micro RNA (miRNA) binding sites. The survival analysis and clinical stage of the target mRNA and corresponding miRNAs were analyzed by LinkedOmics database (http://www.linkedomics.org/ login.php). The study was conducted in accordance with the Declaration of Helsinki (as revised in 2013).

\section{Cell culture}

Human OSCC cell lines TSCC1 (YS-X0472), CAL-27 (klCC101648), HSC-4 (HTX1888), SCC-4 (HS-Cs211105), SCC25 (EY-X1063) and human normal oral mucosal epithelial cells HL-047 (FS-X4623) were purchased from Beijing Boehner Culture Center (Beijing, China). All cell lines were cultured in Dulbecco's modified Eagle medium (DMEM, Sigma-Aldrich, St. Louis, MO, USA) containing $10 \%$ fetal bovine serum (FBS, Gibco, Thermo Fisher Science, Inc., Waltham, MA, USA), $100 \mu \mathrm{g} / \mathrm{mL}$ streptomycin (Gibco, Thermo Fisher Science, Inc.) and $100 \mu \mathrm{g} / \mathrm{mL}$ penicillin (Gibco, Thermo Fisher Science, Inc.) at $37^{\circ} \mathrm{C}$ in a humidified atmosphere of $5 \% \mathrm{CO}_{2}$.

\section{Cellular lentivirus infection}

The lentivirus infection of Mimic NC, miR-29c mimic, shNC, sh-SERPINH1, ov-NC, and ov-SERPINH1 were obtained from Shanghai Genechem Company (Shanghai, China) and the SCC25 cell lines were infected according to the manufacturer's instructions.

\section{$R N A$ extraction and quantitative reverse transcription polymerase chain reaction (RT-qPCR)}

Total RNAs were extracted using Trizol reagent (Invitrogen, Carlsbad, CA, USA) according to the manufacturer's instructions. Reverse transcription (RT) and quantitative RT-qPCR were performed using the PrimeScript RT reagent kit (Takara Bio., Dalian, China) and SYBR Prime Script RT PCR kit (Takara Bio., Dalian, China), respectively. RNU6-1 (U6) and glyceraldehyde 3-phosphate 
Table 1 Primer sequences

\begin{tabular}{ll}
\hline Target gene & Primer (5'-3') \\
\hline miR-29c & F: 5'-GCAGTAGCACCATTTGAAATC-3' \\
& R: 5'-GGTCCAGTTTTTTTTTTTTAACC-3' \\
U6 & F: 5'-CTCGCTTCGGCAGCACA-3' \\
& R: 5'-AACGCTTCACGAATTTGCGT-3' \\
SERPINH1 & F: 5'-CAGAAGTTTCTCGGGACGGG-3' \\
& R: 5'-GCCTGCCTTTTCATTCTGGG-3' \\
GAPDH & F: 5'-GGAGCGAGATCCCTCCAAAAT-3' \\
& R: 5'-GGCTGTTGTCATACTTCTCATGG-3'
\end{tabular}

dehydrogenase (GAPDH) were used as internal control for miR-29c and SERPINH1, respectively. The results were calculated using the $2^{-\Delta \Delta \mathrm{Ct}}$ method. The primer sequences used for RT-qPCR are listed in Table 1.

\section{Western blotting analysis}

Proteins for immunoblotting were resolved by using sodium dodecyl sulfate-polyacrylamide gel electrophoresis (SDSPAGE) and then transferred onto nitrocellulose membrane (Bio-Rad, Hercules, CA, USA). The membrane was blocked with $5 \%$ skimmed milk and then incubated with SERPINH1 rabbit polyclonal antibody (ab109117, Abcam, Cambridge, UK; 1:2,000 dilution; RRID: AB_10888995) and GAPDH rabbit polyclonal antibody ( $" 2118$, Cell Signaling Technology, Boston, MA, USA; 1:5,000; RRID: AB_561053) at room temperature. The membrane was incubated with horseradish peroxidase-conjugated sheep anti-rabbit IgG secondary antibody (ab6721, Abcam; 1:2,000 dilution; RRID: AB_955447) at room temperature for $2 \mathrm{~h}$, and then washed 3 times with phosphate-buffered saline with Tween 20 (PBST, PBS contained 0.1\% Tween 20) for $20 \mathrm{~min}$. Chemiluminescent signal was visualized using a Clarity ${ }^{\mathrm{TM}}$ Western ECL Substrate ( ${ }^{\# 170-5061, \text { Bio- }}$ Rad Laboratories, Inc.,) and detected by Tanon 5200 full automatic chemiluminescence image analysis system (Tanon Science and Technology Co., Ltd., Shanghai, China).

\section{Cell viability and proliferation assay}

Cell viability was measured by 3-(4,5-dimethylthiazol-2yl)-2,5-diphenyl tetrazolium bromide (MTT) assay. At $48 \mathrm{~h}$ after transfection, cells were trypsinized and seeded onto the 96 -well plate $\left(5 \times 10^{3}\right.$ cells per well). After $72 \mathrm{~h}$ of incubation, $10 \mu \mathrm{L}$ of tetrazolium blue reagent $(5 \mathrm{mg} / \mathrm{mL})$ was added to each well and incubated for $4 \mathrm{~h}$ at $37^{\circ} \mathrm{C}$. After the supernatant was removed, $200 \mu \mathrm{L}$ of dimethyl sulfoxide (DMSO) was added, and the resulting colored solution was quantified by measuring the absorbance at the $490 \mathrm{~nm}$ using a mini tablet reader (Bio-Rad Laboratories).

\section{Cell migration assay}

Cell migration ability was determined by wound healing assay and transwell assay. For the wound healing assay, cells were seeded in 6-well plates and transfected with Mimic NC, miR-29c mimic, sh-NC and sh-SERPINH1, ov-NC, or ov-SERPINH1. A linear scratch wound was created on cell monolayers using a sterile $200 \mu \mathrm{L}$ pipette tip, and the scratched area was photographed at $\times 100$ magnification using a Leica DMI3000B computer-assisted microscope (Leica, Buffalo Grove, IL, USA). After scratching, images were captured at $0,24,48$, and $72 \mathrm{~h}$, and the gap area was analyzed using Image-Pro Plus v6.0 image analysis software (Media Cybernetics, Rockville, MD, USA). Transwell assay was performed using transwell chambers (Corning Inc., Corning, NY, USA) with a polycarbonate membrane. Cells $\left(1 \times 10^{5}\right.$ cells) were seeded onto upper transwell insert in serum-free DMEM, and the lower chamber contained $10 \%$ FBS. After incubating for about $10 \mathrm{~h}$, the cells in the upper chambers were wiped off, and the cells in the lower chamber were further stained with crystal violet for $1 \mathrm{~min}$ at $25^{\circ} \mathrm{C}$. The stained cells were observed under a light microscope (Nikon, Melville, NY, USA, 100x) and counted in 5 randomly selected fields.

\section{Cell cycle analysis}

Briefly, the analyzed cells were collected, trypsinized, washed with PBS, and fixed with cold ethanol. The cells were subsequently stained with propidium iodide (PI, Sigma-Aldrich) for $15 \mathrm{~min}$, and the percentage and proportion of cells in G0/G1, S, and G2M phase were analyzed by flow cytometry (Beckman Coulter, Brea, CA, USA).

\section{Dual-luciferase reporter assay}

For dual-luciferase reporter assay, the 3 prime untranslated region (3'-UTR) of SERPINH1 containing wild-type or mutated putative miR-29c binding site was constructed. 
The SCC25 cells were cultured in 24-well plates and then co-transfected with the above luciferase reporter plasmids and miR-29c mimic (or mimic NC for control group) using Lipofectamine 2000 reagent (Invitrogen). After transfection for $48 \mathrm{~h}$, the cell lysates were collected and luciferase activity was detected by dual luciferase reporter gene detection system (Promega, Madison, WI, USA).

\section{Statistical analysis}

All statistical data were analyzed using SPSS 20.0 software (IBM Corp., Chicago, IL, USA). All data were presented as mean \pm standard deviation (SD). The difference between groups was analyzed by $t$-test. Pearson chi-square $\left(\chi^{2}\right)$ test was used to analyze the relationship between expression of miR-29c and SERPINH1. All experiments in the study were independently repeated 3 times, which were technical replicates. A $\mathrm{P}$ value $<0.05$ or $<0.01$ was considered statistically significant.

\section{Results}

\section{The high expression of SERPINH1 in OSCC is related to} poor prognosis

As shown in Figure $1 A, 1 B$, the gene expression in each sample was symmetrically distributed with other samples. The median-centered values in all samples were indicative, thus the samples were normalized and cross-comparable. To paraphrase, subsequent bioinformatics analysis could be continued, and the data was valuable for further screening. Through the analysis of GEO2R in the GSE31056 dataset, we found that SERPINH1 was highly expressed in tumor tissues (Figure 1C). In addition, the analysis of the UALCAN database further showed that SERPINH1 was highly expressed in OSCC patients (Figure 1D), and patients with high SERPINH1 expression were significantly associated with poor survival (Figure 1E). Combined with the clinical data of the patients in the UALCAN database, there were significant differences between individual cancer (Figure $1 F$ ), tumor grade (Figure $1 G$ ) and nodal metastasis status (Figure 1H) of OSCC, and the expression level of SERPINH1 increased with the increase of tumor stages. Furthermore, the expression of SERPINH1 in the OSCC cell line was significantly higher than that in the human normal oral mucosal epithelial cell line HL-047 (Figure 1I,17). Taken together, SERPINH1 may play an oncogenic role in OSCC, and its high expression is associated with poor prognosis.

\section{Down-regulation of SERPINH1 inbibited the proliferation and migration and promoted cell cycle arrest and apoptosis in OSCC cell line}

The mRNA and protein levels of SERPINH1 in SCC25 cells transfected with sh-SERPINH1 were significantly decreased (Figure 2A,2B). The results of MTT assay showed that after inhibiting the expression of SERPINH1, the cell viability of SCC25 cells decreased significantly (Figure 2C). The results of wound-healing and transwell assays showed that the decreased expression of SERPINH1 reduced the migration ability of SCC25 cells (Figure 2D,2E). The results of 4',6-diamidino-2-phenylindole (DAPI) staining further showed that down-regulation of SERPINH1 expression reduced cell proliferation and promoted apoptosis (Figure $2 F$ ). The results of cell cycle analysis showed that the proportion of G0/G1 phase cells in the shSERPINH1 group was significantly lower than that in the control group, while the proportion of $\mathrm{S}$ phase cells was significantly higher than that in the sh-SERPINH1 group (Figure 2G). These results indicate that the reduced SERPINH1 expression inhibited the proliferation of OSCC tumor cells.

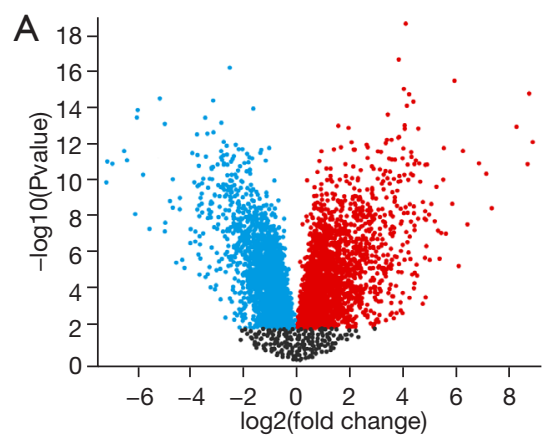

(c) Annals of Translational Medicine. All rights reserved.

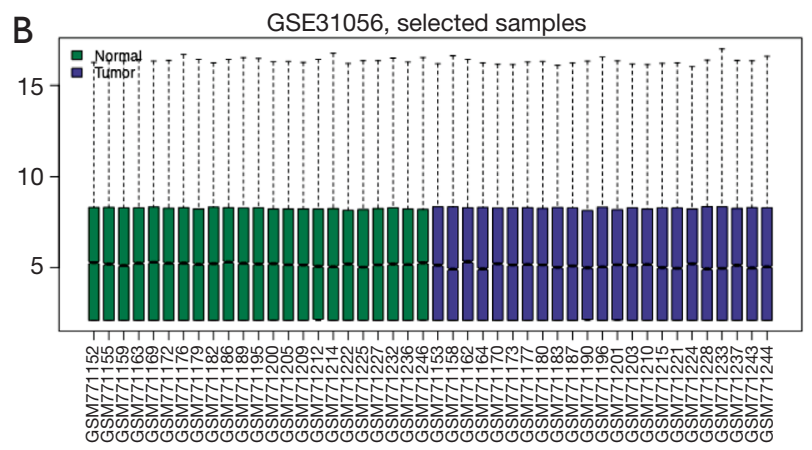

Ann Transl Med 2021;9(18):1423 | https://dx.doi.org/10.21037/atm-21-3720 


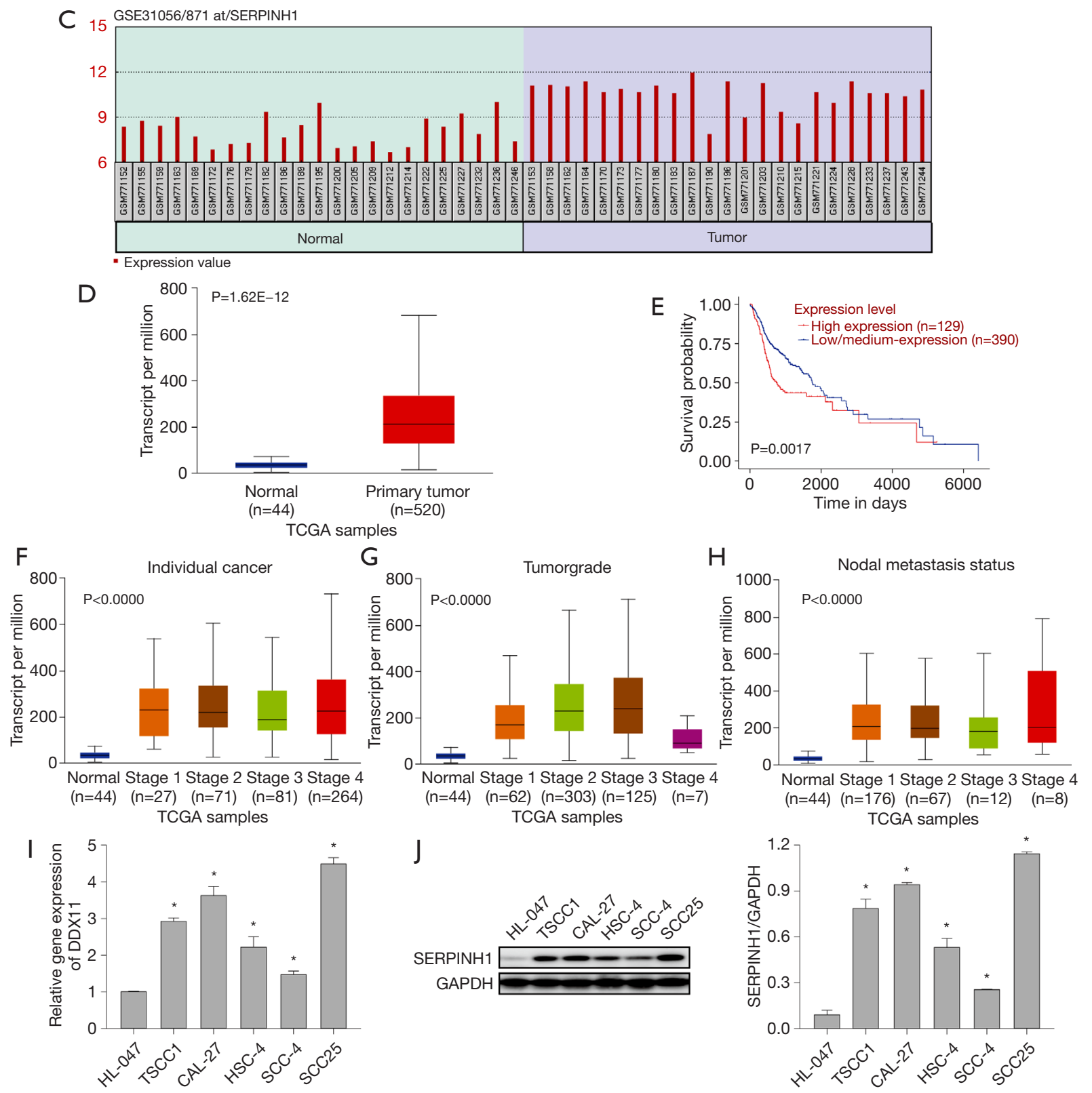

Figure 1 Expression profile and prognosis of SERPINH1 in OSCC. (A) The volcano map of GSE31056 dataset of differently expressed genes between normal and tumor tissues. (B) The box diagram of the distribution of the values of the 23 para-cancerous tissues and 23 OSCC tumor tissues. (C,D) GEO database. The low expression of SERPINH1 in normal tissues (blue) and the high expression of SERPINH1 in tumor tissues (red). (E) Survival probability of SERPINH1 expression based on UALCAN database. Red line indicates patients with high expression of SERPINH1, and blue line indicates patients with low expression of SERPINH1. (F-H) Box map shows the expression of SERPINH1 in different individual cancer stages, clinicopathological grades, and lymph node metastatic status stages of OSCC. (I,J) SERPINH1 mRNA and protein levels in HL-047 and OSCC cell lines (TSCC1, CAL-27, HSC-4, SCC-4, SCC25). *, P<0.05 vs. HL-047. OSCC, oral squamous cell carcinoma; GEO, Gene Expression Omnibus; UALCAN, public cancer transcriptome database; mRNA, messenger RNA. 

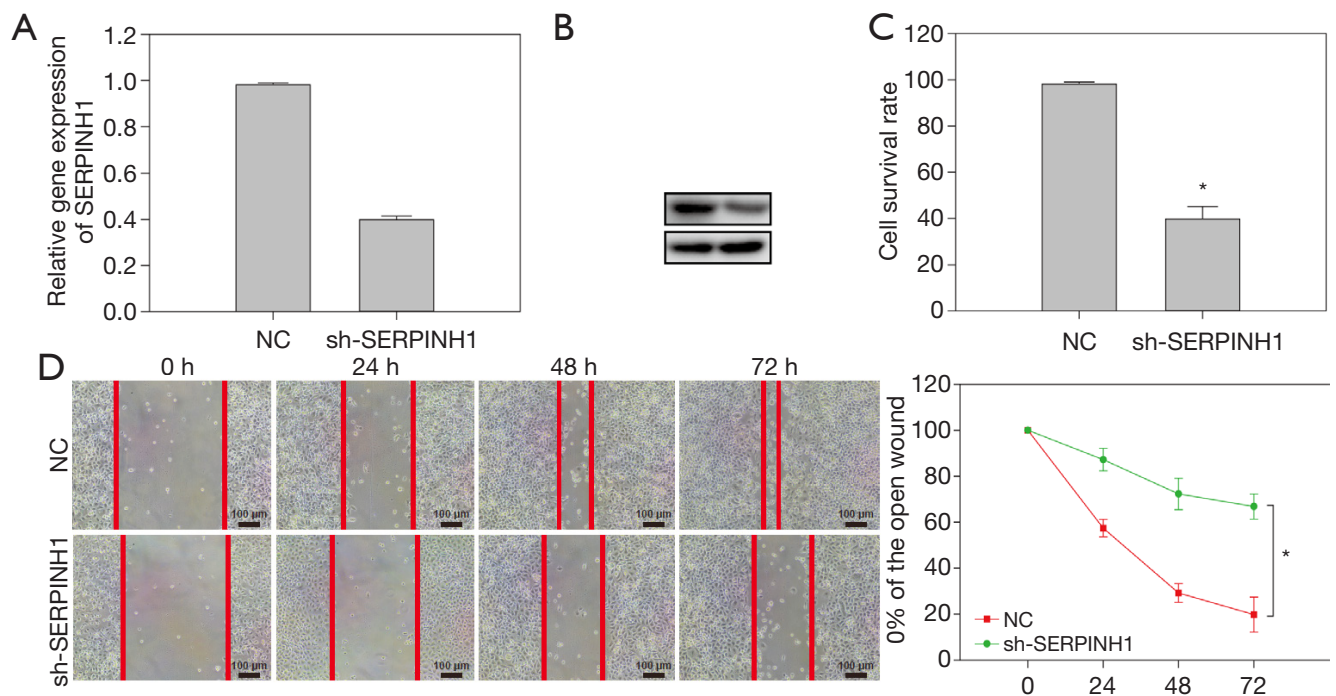

$72 \mathrm{~h}$

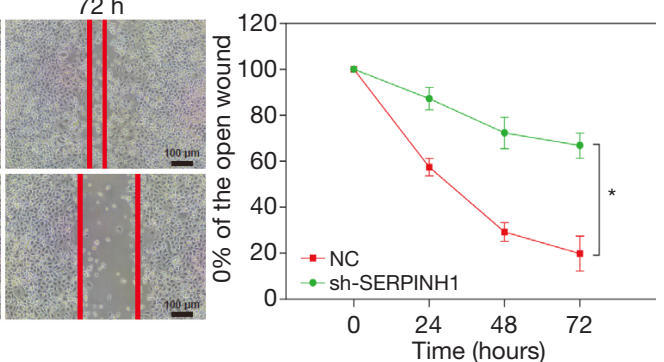

$E$

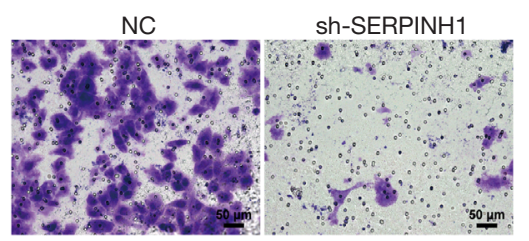

$\mathrm{F}$
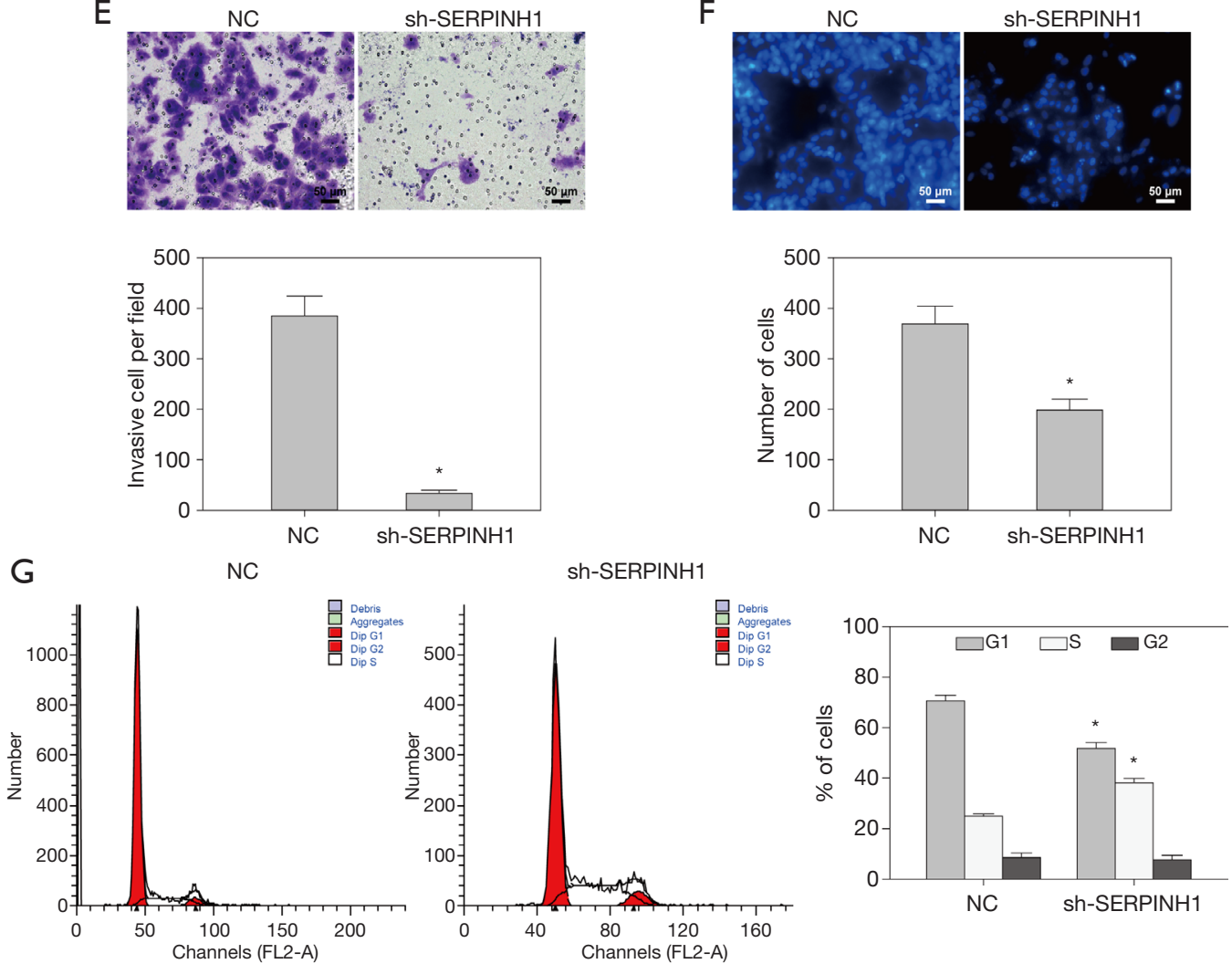

Figure 2 The low expression of SERPINH1 inhibits the proliferation and migration of OSCC cells and promotes cell cycle arrest. (A,B) SERPINH1 mRNA and protein levels in OSCC cells before and after silencing the expression of SERPINH. (C) MTT colorimetric assay of the cell viability of OSCC cells after silencing the expression of SERPINH1 for $72 \mathrm{~h}$. (D) Wound-healing assay and (E) Transwell assay were used to measure the migration ability of OSCC cells. (F) DAPI staining was used to examine the apoptosis of OSCC cells. (G) Flow cytometry was used to analyze the cell cycle state of OSCC cells. *, $\mathrm{P}<0.05$ vs. NC. OSCC, oral squamous cell carcinoma; mRNA, messenger RNA; MTT, 3-(4,5-dimethylthiazol-2-yl)-2,5-diphenyl tetrazolium bromide; DAPI, 4',6-diamidino-2-phenylindole. 


\section{SERPINH1 as the downstream target gene of miR-29c}

In order to understand the underlying regulatory mechanism of SERPINH1 in OSCC, we excavated and analyzed its upstream regulatory factors. TargetScan database was used to analyze the putative binding miRNAs on SERPINH1 and identified that miR-29c may act as the potential regulator of SERPINH1 (Figure $3 A$ ). LinkedOmics database analysis of miR-29c showed that there were differences among different clinicopathological grades (Figure 3B), different individual cancer stages (Figure 3C), and lymph node metastatic status stages (Figure 3D). In addition, LinkedOmics database survival analysis showed that patients with high miR-29c expression were associated with better survival outcome than patients with low miR-29c expression (Figure 3E). Pearson correlation analysis further showed that there was a negative correlation between miR29c and SERPINH1 (Figure 3F). In addition, he expression of miR-29c in OSCC cell line was significantly lower than that in HL-047 cell line (Figure 3G), while the expression of SERPINH1 in these 2 cell lines was opposite. In order to validate the targeted regulation relationship between miR-29c and SERPINH1, we first examined the mRNA and protein expression of SERPINH1 in miR-29c mimictransfected OSCC cells. The results showed that the expression of SERPINH1 was significantly down-regulated in miR-29c overexpressed cells (Figure 3H,3I). The results of dual luciferase reporter assay showed that miR-29c exerted a significant inhibitory effect on luciferase activity of the SERPINH1-WT group (Figure 37). Taken together, these results indicate that SERPINH1 is the downstream target of miR-29c and is negatively regulated by miR-29c in OSCC.

\section{Overexpression of miR-29c inbibits the proliferation and migration and promotes cell cycle arrest and apoptosis in OSCC cells}

Since miR-29c can regulate the expression of SERPINH1, we further studied the effect of miR-29c overexpression on cell function. The transfection efficiency of miR$29 \mathrm{c}$ is shown in Figure $4 A-4 C$. The results of MTT assay (Figure $4 D$ ), wound healing assay (Figure $4 E$ ), transwell assay (Figure $4 F$ ), and DAPI staining (Figure $4 G$ ) showed that miR-29c overexpression significantly inhibited the proliferation and migration of OSCC cells and promoted apoptosis. Moreover, the overexpression of SERPINH1 significantly reduced the inhibitory effects of miR-29c on cell migration, invasion, and apoptosis. In addition, overexpression of miR-29c caused cell cycle arrest at S phase, which may be the cause of the inhibition of OSCC cell proliferation (Figure 4H). In summary, these results indicate that miR-29c overexpression can inhibit the cellular function of OSCC, and SERPINH1 overexpression can reverse the inhibitory effect of miR-29c.

\section{Discussion}

In the present study, we found that the expression of SERPINH1 was significantly up-regulated in OSCC. Cell biology experiments showed that silencing SERPINH1 expression inhibited the proliferation and metastasis of OSCC cells. Mechanism studies further showed that miR$29 \mathrm{c}$ can regulate the expression of SERPINH1. In addition, bioinformatics analysis of the GSE31056 dataset revealed that the expression of SERPINH1 was significantly upregulated in OSCC tumor tissues. At the same time, this study also confirmed the increased mRNA and protein expression of SERPINH1 in OSCC cell lines.

Another bioinformatics analysis supported our finding that the expression of SERPINH1 was significantly increased in HNSCC and associated with poor overall survival (OS) rate, indicating that SERPINH1 is a potential clinical target and prognostic marker of HNSCC (11). Genome-wide analysis of alternative splicing revealed the specific expression of SERPINH1 in human hepatocellular carcinoma (12), and another study also showed that the expression of SERPINH1 is related to the development and prognosis of pancreatic ductal adenocarcinoma (13). Therefore, SERPINH1 may play an important role in OSCC tumorigenesis and development. In this study, the UALCAN database was used to examine the association between the expression of SERPINH1 and the survival and clinical staging of OSCC patients. We found that high SERPINH1 expression is significantly associated with poor survival. In addition, the expression of SERPINH1 differed significantly in different individual cancer stages, different clinicopathological grades, and different lymph node metastasis stages $(4,9)$. Thus, we recommend using SERPINH1 as a prognostic marker for survival and early diagnosis of OSCC. In addition, we also verified the effect of SERPINH1 on cell function by silencing the expression of SERPINH1 in SCC25 cells, and found that silencing SERPINH1 not only significantly inhibited cell proliferation and migration, but also significantly increased the proportion of cells in S phase. Thus, our study further 


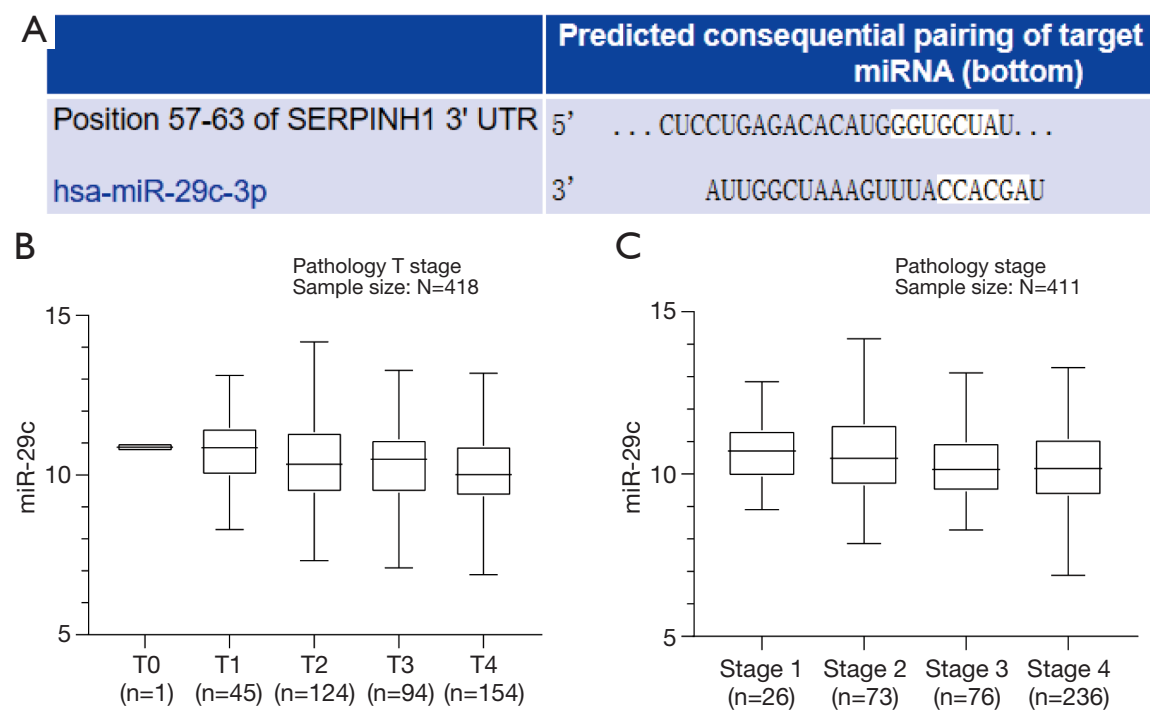

Site Context+ type score

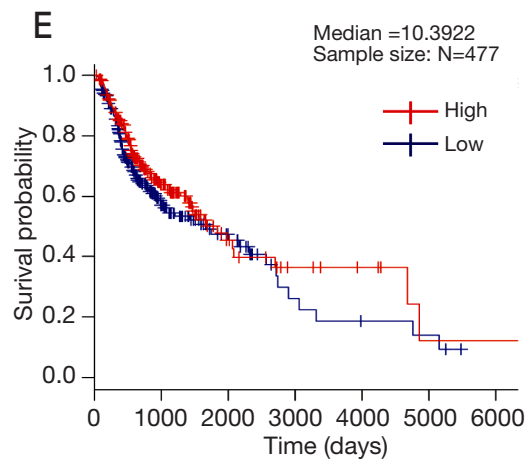

$\mathrm{H}$

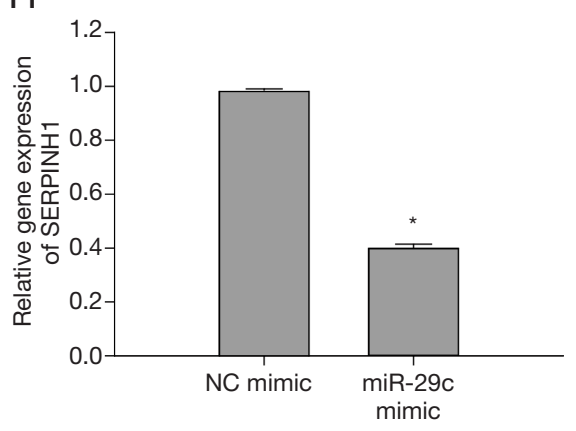

$\mathrm{F}$

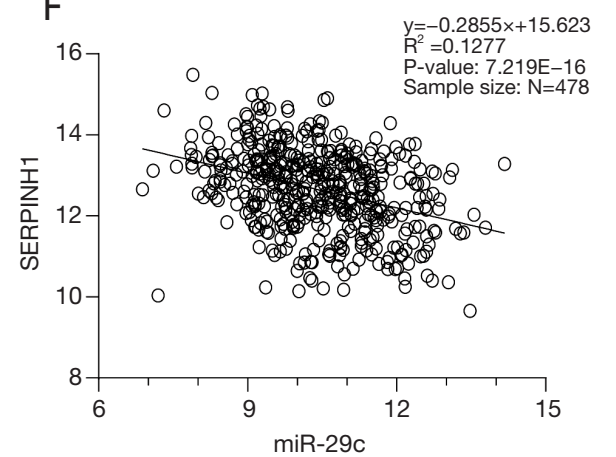

I

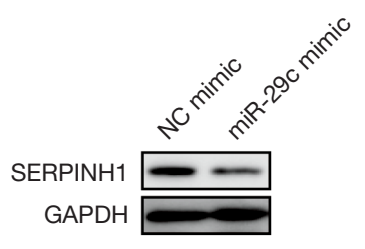

7mer-

A1 -0.28

D

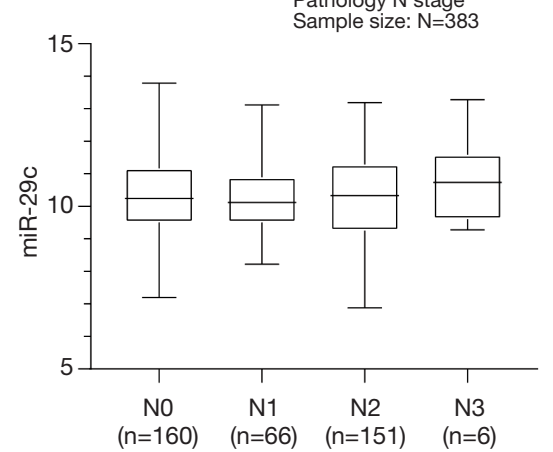

G

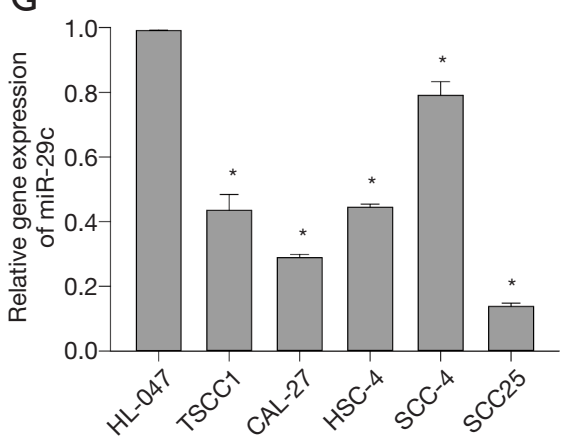

$\mathrm{J}$

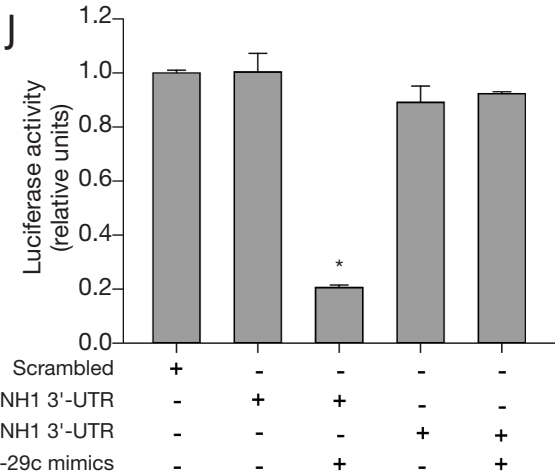

Figure 3 Overexpression of miR-29c down-regulates the expression of SERPINH1. (A) TargetScan analysis predicted the putative miR$29 \mathrm{c}$ binding sites on the 3'-UTR of SERPINH1. Results of LinkedOmics database analysis of the expression profile of miR-29c in different clinicopathological grades (B), individual cancer stages (C), and lymph node metastasis stages (D). (E) Survival probability of miR-29c expression in 477 patients with OSCC. Red line denotes patients the high miR-29c expression, while blue line represents patients with low miR-29c expression. (F) Pearson correlation analysis of the relationship between SERPINH1 expression and miR-29c expression. (G) Relative miR-29c expression in HL-047, TSCC1, CAL-27, HSC-4, SCC-4, and SCC25 cells. (H) Overexpression of miR-29c suppressed the mRNA level of SERPINH1. (I) Overexpression of miR-29c suppressed the protein level of SERPINH1. (J) Dual luciferase reporter assay was used to determine the targeted binding of miR-29c and SERPINH1. Differences were found to be statistically significant at *, $\mathrm{P}<0.05$. 3'-UTR, 3 prime untranslated region; OSCC, oral squamous cell carcinoma. 

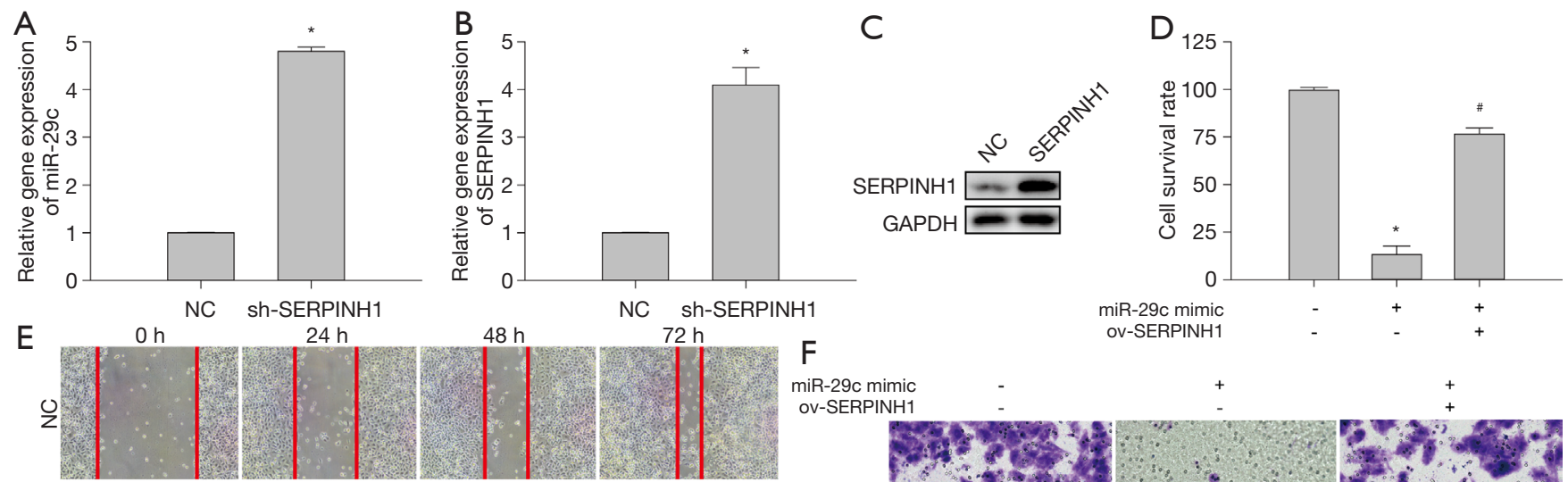

F ov-SERPINH1
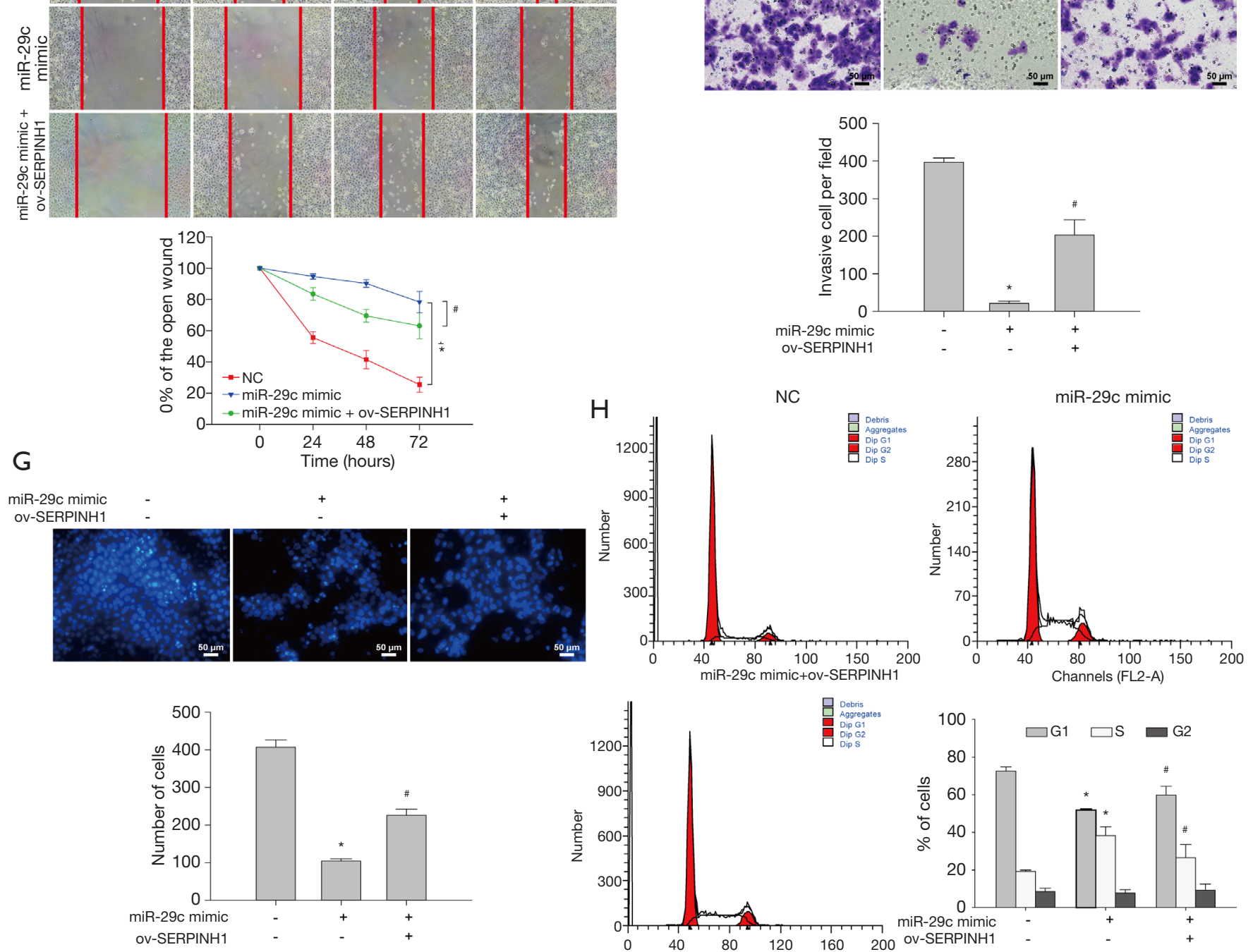

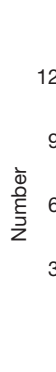

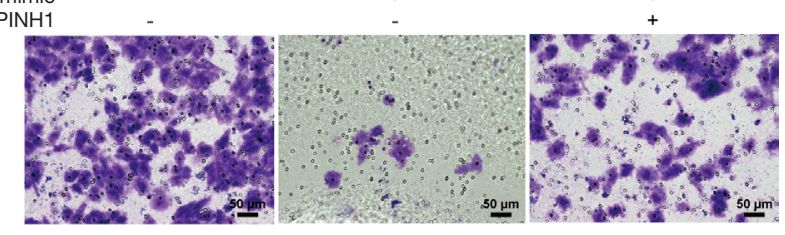
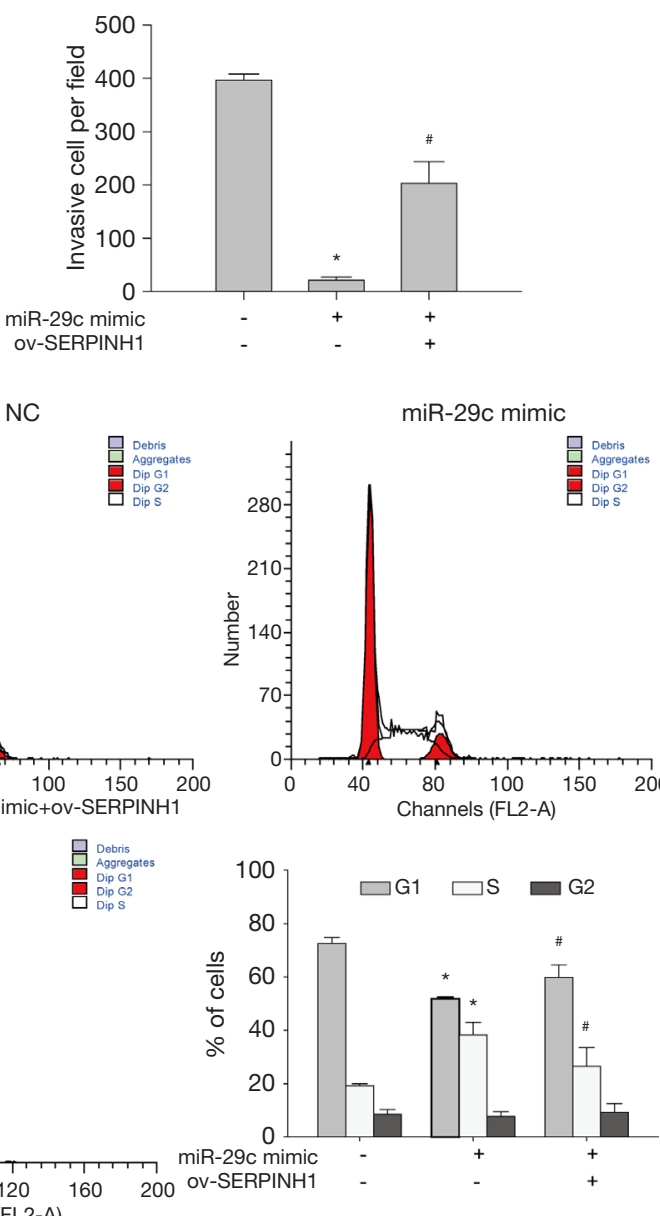

Figure 4 SERPINH1 overexpression reversed the inhibitory effects of miR-29c on OSCC proliferation, migration, apoptosis, and cell cycle arrest. (A) Relative expression level of miR-29c after transfection of miR-29c mimic. (B,C) Relative mRNA and protein levels of SERPINH1 in OSCC cells after SERPINH1 overexpression. (D) The viability of OSCC cells at $72 \mathrm{~h}$ was detected by MTT colorimetric assay. (E,F) The migration and invasion ability of OSCC cells was measured by wound healing assay and transwell assay, respectively. (G) The apoptosis of OSCC cells was detected by DAPI staining. (H) The proportion of cells in G1, S, and G2 phases was analyzed by flow cytometry. *, $\mathrm{P}<0.05$ vs. NC; ${ }^{\#}, \mathrm{P}<0.05$ vs. miR-29c mimic. OSCC, oral squamous cell carcinoma; mRNA, messenger RNA; MTT, 3-(4,5-dimethylthiazol2-yl)-2,5-diphenyl tetrazolium bromide; DAPI, 4',6-diamidino-2-phenylindole. 
clarified the crucial function of SERPINH1 in OSCC.

It is known that miRNAs plays an important role in biological processes, such as cell migration, differentiation, and apoptosis, as well as chemoresistance of tumor cells (14). The miRNAs can directly bind to the target gene mRNA to cause translational inhibition or post-transcriptional degradation of targeted mRNA (15). In order to further explore the upstream regulation mechanism of SERPINH1, we analyzed the TargetScan and LinkedOmics databases through bioinformatics, and found that miR-29c may be putative binding site located on the 3'-UTR of SERPINH1 mRNA. A series of experimental analyses showed that miR-29c can specifically down-regulate the expression of SERPINH1 in OSCC cells, suggesting that miR-29c may act as a tumor suppressor to regulate the tumorigenicity of OSCC (13). A study by Lopes et al. showed that compared to non-cancerous tissues of healthy volunteers, miR$29 \mathrm{c}$ is significantly expressed in oral cancer tissues and adjacent normal tissues (16). The miR-29c-KIAA1199 axis regulates gastric cancer migration by binding with WBP11 and PTP4A3 (17). In addition, miR-29c can inhibit the expression of FBXO31 by directly binding to the 3'UTR of FBXO31, resulting in downstream activation of p38 MAPK and subsequent 5-FU chemoresistance of esophageal squamous cell carcinoma (ESCC) (18). Up-regulation of long non-coding RNA TUG1 can inhibit miR-29c and subsequently promotes the proliferation, migration, and invasion of bladder cancer cells (19). In breast cancer cells, miR-29c acts as a tumor suppressor miRNA by targeting the TIMP3/STAT1/FoxO1 signaling pathway (20). In order to further verify whether miR-29c also plays a tumor suppressor in the tumorigenesis of OSCC, we overexpressed miR-29c in OSCC cell lines, and found that miR-29c overexpression significantly inhibited the proliferation and metastasis of OSCC cells. Moreover, when SERPINH1 was further overexpressed, the tumor suppressor effect of miR$29 \mathrm{c}$ was reversed, which is consistent with the role of miR$29 \mathrm{c}$ in other tumors. It is suggested that miR-29c may play an important role in tumor treatment. In the future, the miR-29c/SERPINH1 axis may be used as a therapeutic to improve the therapeutic effect and prognosis of OSCC.

\section{Conclusions}

An important role is played by SERPINH1 in the proliferation and migration of cancer cells. This study explored the mechanism by which miR-29c regulates the expression of SERPINH1 in the malignant process of
OSCC. We found that SERPINH1 can not only be used as a marker for OSCC diagnosis, but also can be used as therapeutic target to improve survival of patients with OSCC. This may become a new direction for our future research. In conclusion, this study has provided a new theoretical basis for future molecular targeted therapy of OSCC.

\section{Acknowledgments}

Funding: This study was supported by the grants of Liaoning Province Key Scientific and Technological Project (20180550035).

\section{Footnote}

Reporting Checklist: The authors have completed the MDAR reporting checklist. Available at https://dx.doi. org/10.21037/atm-21-3720

Conflicts of Interest: All authors have completed the ICMJE uniform disclosure form (available at https://dx.doi. org/10.21037/atm-21-3720). All authors have completed the ICMJE uniform disclosure form. CW reports funding support from the Liaoning Province Key Scientific and Technological Project (20180550035). The authors have no other conflicts of interest to declare.

Ethical Statement: The authors are accountable for all aspects of the work in ensuring that questions related to the accuracy or integrity of any part of the work are appropriately investigated and resolved. The study was conducted in accordance with the Declaration of Helsinki (as revised in 2013).

Open Access Statement: This is an Open Access article distributed in accordance with the Creative Commons Attribution-NonCommercial-NoDerivs 4.0 International License (CC BY-NC-ND 4.0), which permits the noncommercial replication and distribution of the article with the strict proviso that no changes or edits are made and the original work is properly cited (including links to both the formal publication through the relevant DOI and the license). See: https://creativecommons.org/licenses/by-nc-nd/4.0/.

\section{References}

1. Bray F, Ferlay J, Soerjomataram I, et al. Global cancer 
statistics 2018: GLOBOCAN estimates of incidence and mortality worldwide for 36 cancers in 185 countries. CA Cancer J Clin 2018;68:394-424.

2. Roi A, Roi CI, Negruțiu ML, et al. The Challenges of OSCC Diagnosis: Salivary Cytokines as Potential Biomarkers. J Clin Med 2020;9:2866.

3. Yadav KD, Patil BA, Raheel SA, et al. Serum uric acid levels in patients with oral cancer, leukoplakia and submucous fibrosis: a cross-sectional study. Transl Cancer Res 2020;9:3084-91.

4. Tian S, Peng P, Li J, et al. SERPINH1 regulates EMT and gastric cancer metastasis via the $\mathrm{Wnt} / \beta$-catenin signaling pathway. Aging (Albany NY) 2020;12:3574-93.

5. Wu G, Ju X, Wang Y, et al. Up-regulation of SNHG6 activates SERPINH1 expression by competitive binding to $\mathrm{miR}-139-5 \mathrm{p}$ to promote hepatocellular carcinoma progression. Cell Cycle 2019;18:1849-67.

6. Kamikawaji K, Seki N, Watanabe M, et al. Regulation of LOXL2 and SERPINH1 by antitumor microRNA-29a in lung cancer with idiopathic pulmonary fibrosis. J Hum Genet 2016;61:985-93.

7. Yamada Y, Sugawara S, Arai T, et al. Molecular pathogenesis of renal cell carcinoma: Impact of the antitumor miR-29 family on gene regulation. Int J Urol 2018;25:953-65.

8. Kawagoe K, Wada M, Idichi T, et al. Regulation of aberrantly expressed SERPINH1 by antitumor miR-148a$5 \mathrm{p}$ inhibits cancer cell aggressiveness in gastric cancer. J Hum Genet 2020;65:647-56.

9. Qi Y, Zhang Y, Peng Z, et al. SERPINH1 overexpression in clear cell renal cell carcinoma: association with poor clinical outcome and its potential as a novel prognostic marker. J Cell Mol Med 2018;22:1224-35.

10. Barrett T, Wilhite SE, Ledoux P, et al. NCBI GEO: archive for functional genomics data sets--update. Nucleic Acids Res 2013;41:D991-5.

11. Fan $\mathrm{G}, \mathrm{Tu} \mathrm{Y}, \mathrm{Wu} \mathrm{N}$, et al. The expression profiles and

Cite this article as: Wang C, Wang Z, Zhang L, Lin X. MiR$29 \mathrm{c}$ inhibits the metastasis of oral squamous cell carcinoma and promotes its cell cycle arrest by targeting SERPINH1. Ann Transl Med 2021;9(18):1423. doi: 10.21037/atm-21-3720 prognostic values of HSPs family members in Head and neck cancer. Cancer Cell Int 2020;20:220.

12. Chen H, Gao F, He M, et al. Long-Read RNA Sequencing Identifies Alternative Splice Variants in Hepatocellular Carcinoma and Tumor-Specific Isoforms. Hepatology 2019;70:1011-25.

13. Zhang Y, Zhu L, Wang X. A Network-Based Approach for Identification of Subtype-Specific Master Regulators in Pancreatic Ductal Adenocarcinoma. Genes (Basel) 2020;11:155.

14. Vishnubalaji R, Shaath H, Elango R, et al. Noncoding RNAs as potential mediators of resistance to cancer immunotherapy. Semin Cancer Biol 2020;65:65-79.

15. Mei D, Tan WSD, Tay Y, et al. Therapeutic RNA Strategies for Chronic Obstructive Pulmonary Disease. Trends Pharmacol Sci 2020;41:475-86.

16. Lopes CB, Magalhães LL, Teófilo CR, et al. Differential expression of hsa-miR-221, hsa-miR-21, hsa-miR-135b, and hsa-miR-29c suggests a field effect in oral cancer. BMC Cancer 2018;18:721.

17. Wang L, Yu T, Li W, et al. The miR-29c-KIAA1199 axis regulates gastric cancer migration by binding with WBP11 and PTP4A3. Oncogene 2019;38:3134-50.

18. Li B, Hong P, Zheng CC, et al. Identification of miR-29c and its Target FBXO31 as a Key Regulatory Mechanism in Esophageal Cancer Chemoresistance: Functional Validation and Clinical Significance. Theranostics 2019;9:1599-613.

19. Guo P, Zhang G, Meng J, et al. Upregulation of Long Noncoding RNA TUG1 Promotes Bladder Cancer Cell Proliferation, Migration, and Invasion by Inhibiting miR29c. Oncol Res 2018;26:1083-91.

20. Li W, Yi J, Zheng X, et al. miR-29c plays a suppressive role in breast cancer by targeting the TIMP3/STAT1/ FOXO1 pathway. Clin Epigenetics 2018;10:64.

(English Language Editor: J. Jones) 